\title{
Single-Blind Study of Dystrophin Staining in Carriers of Duchenne Muscular Dystrophy
}

\author{
Francois P. Bernier, Cheryl R. Greenberg, William C. Halliday and Klaus Wrogemann
}

\begin{abstract}
A single-blind study of dystrophin staining in skeletal muscle was performed in 13 biopsies from carriers of Duchenne Muscular Dystrophy (DMD) and controls. The results indicate that immunohistochemical analysis of dystrophin staining is a valuable diagnostic test for DMD carriers when DNA for testing is unavailable from critical family members or is uninformative, when creatine kinase (CK) values are conflicting or when $\mathrm{CK}$ values must be used in isolation.
\end{abstract}

RÉSUMÉ: Coloration de la dystrophine chez les porteurs de la dystrophie musculaire de Duchenne. Nous avons procédé à une étude en simple insu de la coloration de la dystrophine dans des biopsies de muscles squelettiques provenant de porteurs de la dystrophie musculaire de Duchenne (DMD) et chez des contrôles. Les résultats indiquent que l'analyse immunohistochimique par coloration de la dystrophine est une épreuve diagnostique valable chez les porteurs de la DMD quand I'ADN de certains membres stratégiques de la famille n'est pas disponible ou n'est pas informatif, quand les valeurs de créatine-kinase $(\mathrm{CK})$ sont discordantes ou quand les valeurs de CK doivent être utilisées de façon isolée.

Can. J. Neurol. Sci. 1993; $20: 44-47$

With $66 \%$ of mothers of children with Duchenne Muscular Dystrophy predicted to be carriers, the ability to definitively assign carrier status is essential to ensure accurate genetic counselling.' A review of our local five year experience of carrier studies in DMD revealed that in spite of remarkable advances in DNA testing, definitive ( $>95 \%$ or $<5 \%$ probability) carrier assignment was still not possible in 4 of $35(11 \%)$ DMD families studied because either critical DNA samples were unavailable or DNA analysis was uninformative. Furthermore, $35 \%$ of mothers of "sporadic" DMD boys are still being counselled as high probability carriers on the basis of CK data and Bayesian analysis alone.

Immunohistochemical analysis of dystrophin expression has been previously reported as a means of direct carrier detection. ${ }^{2 \cdot 7}$ Although reports have confirmed the presence of a mosaic pattern in some carriers, the worldwide experience is limited and controversy still exists over the sensitivity and specificity of dystrophin immunohistochemistry as a direct diagnostic test. As dystrophin-negative fibres appear to be rarely seen in asymptomatic carriers, ${ }^{8.9}$ we felt it was important to conduct this study free from potential bias in a single-blind fashion.

The results of this single blind study suggest that dystrophin immunohistochemistry may indeed be useful as an ancillary test for DMD carrier assignment and that the specificity is sufficiently high for the test to be used in certain diagnostic situations, particularly when DNA results are unavailable or uninformative.

\section{Materials AND Methods}

The study pool consisted of open quadriceps muscle biopsies obtained from seven normal individuals and six carrier females each of whom had at least one son with well documented DMD. The seven normal biopsies (histology and histochemistry) were obtained from patients undergoing investigations for malignant hyperthermia. Their caffeine-halothane contracture tests were normal. The carrier biopsies were obtained from three obligate carriers (either 2 affected sons or at least one affected son and one affected brother) and three high probability carriers (one symptomatic female and two with detectable DNA alterations). Informed consent was obtained prior to biopsy, and the study was approved by the Faculty Committee on the Use of Human Subjects in Research. Muscle biopsies were snap frozen in liquid nitrogen cooled 2-methyl-butane and stored at $-70^{\circ} \mathrm{C}$. The biopsies were randomly coded by a third party. All biopsies were processed using two mouse monoclonal anti-dystrophin antibodies (Nova Castra, Newcastle), Dys 1, directed against amino acids 1181-1388 in the rod portion of dystrophin and Dys-2, directed against the last 12 amino acids at the carboxy terminus. Both peroxidase and immunofluorescence techniques were used according to the following protocol.

Eight micron frozen sections were prepared on amino-alkyl silane (AAS) coated slides. Dys- 1 and Dys-2 were used undiluted and incubated overnight and 2 hours respectively. The

From the Departments of Pediatrics \& Child Health (F.C.B., C.R.G.), Human Genetics (F.C.B., C.R.G., K.W.), Pathology (F.C.B., W.C.H.) and Biochemistry and Molecular Biology (F.C.B., K.W.), University of Manitoba, Winnipeg

Received April 22, 1992. Accepted in final form September 22, 1992

Reprint requests to: Dr. K. Wrogemann, Department of Biochemistry and Molecular Biology, University of Manitoba, Winnipeg, Manitoba, Canada R3E 0W3 
second antibody was biotinylated anti-mouse (Biogenex, SanRamon, CA, streptavidin kit) for the peroxidase slides and biotinylated anti-mouse (Amersham, Arlington Heights, IL) $1 / 100$ in $0.1 \%$ bovine serum albumin (BSA) diluted in phosphate buffered saline (PBS) for the immunofluorescence. For all the peroxidase slides, streptavidin linked peroxidase (Biogenex kit) was used undiluted and developed using diaminobenzamine (DAB) PBS $\mathrm{H}_{2} \mathrm{O}_{2}$, counterstained and mounted. For immunofluorescence, streptavidin Texas Red (Amersham) was used 1/100 in PBS. As negative controls, both the primary antibody was omitted and biopsies from known DMD males were used.

Individual fibres were scored as positive if the staining was continuous around the fibre, uncertain if the fibre appeared incompletely stained or negative if no staining was visible at the sarcolemma. In total, three hundred fibres were scored for each slide (total 23,400 fibres) using random non-overlapping fields at $200 \times$ magnification. On all biopsies, the fibres at the periphery of the biopsy were not scored. A standard pathology report was independently prepared by one of us (WCH) based on studies of paraffin sections as well as a battery of standard histochemical stains and reactions.

The biopsies were scored blindly. The investigator did not even know the proportion of carriers and controls. Prior to breaking the code, all 13 women in the study were assigned either a low probability or a high probability of being a carrier for DMD based on the presence or absence of a mosaic staining pattern in their biopsies.

\section{Results}

Based on the presence of dystrophin negative fibres and prior to breaking the code, four subjects, $(\# 2,10,11,13)$ were assigned a high probability of being a DMD carrier due to the presence of a mosaic staining pattern and nine subjects were assigned a low probability of being a carrier. Breaking the code revealed all four subjects, predicted to be carriers based on their dystrophin staining pattern, were in fact known carriers of DMD. The seven normal controls in our study were also correctly assigned. However, the two-remaining known carriers of DMD were misclassified as non-carriers and thus represent false-negatives (Table 1).
In known affected DMD patients, no positive staining was observed in any fibres and omission of the primary antibody as a negative control consistently resulted in completely negative slides. In the four biopsies with a mosaic pattern (Figure 1), the percentage of fibres with no staining at the sarcolemmal membrane ranged from $0.7 \%$ to $10.9 \%$ with the only symptomatic carrier (\#13) having the highest proportion of negative tibres. The negative fibres were distributed throughout the biopsies and occurred both in isolation and also in small clusters. The two remaining carrier biopsies were indistinguishable from the normal controls. Using the Fisher exact test, a $98 \%$ correlation existed between the presence of negative fibres and being a carrier for DMD $(p<0.025)$.

Both antibodies with both techniques resulted in a low percentage of fibres which were scored as uncertain (data not shown). On the average, $1.8 \%$ of the fibres were scored uncertain with the range being $0-18 \%$.

There were no neuropathological abnormalities in $4 / 6$ carrier biopsies. Subject 11 's biopsy showed mild, non-specific changes consisting of internal nuclei and a mild increase in the spectrum of fibre sizes. Subject 13, in addition to the pathology noted for Subject 11, had increased endomysial connective tissue and fibre splitting. Both of these carriers also had negatively staining fibres ( $5 \%$ and $10 \%$ respectively). None of the biopsies showed necrotic fibres.

\section{Discussion}

Women carry two copies of the X-chromosome and therefore two doses of $X$-linked genes. Random $X$-inactivation (Lyonization) restores the gene dosage to a single copy per nucleus. ${ }^{10}$ This random process, which occurs early during embryogenesis, results in women who are somatic mosaics for $\mathrm{X}$-linked genes. DMD carrier muscle cells, which develop from the random fusion of myoblasts, will contain both dystrophincompetent and dystrophin-incompetent nuclei. The presence of dystrophin-competent nuclei can therefore account for the observation that the majority or all of the muscle fibres in carriers appear to have normal dystrophin staining. Several processes and factors can explain the variability in the proportion of negative fibres observed in adult carriers. These include: skewed

Table 1. Data from All Carrier and Control Subjects

\begin{tabular}{|c|c|c|c|c|c|c|c|c|c|}
\hline \multirow[b]{3}{*}{ Subject } & \multirow[b]{3}{*}{ Carrier Status* } & \multirow[b]{3}{*}{$\mathrm{CK}^{+}(\mathrm{U} / \mathrm{L})$} & \multirow[b]{3}{*}{ Symptoms } & \multirow[b]{3}{*}{ Pathology } & \multirow[b]{3}{*}{ Age (yrs) ${ }^{+}$} & \multicolumn{4}{|c|}{$\begin{array}{c}\text { Number of Negative } \\
\text { Fibres } / \mathbf{3 0 0}\end{array}$} \\
\hline & & & & & & \multicolumn{2}{|c|}{$\begin{array}{c}\text { Immuno- } \\
\text { fluorescence }\end{array}$} & \multicolumn{2}{|c|}{ Peroxidase } \\
\hline & & & & & & Dys! & Dys2 & Dys1 & Dys2 \\
\hline 2 & $\mathrm{H}$ & 797 & No & No & 28 & 0 & 2 & 0 & 0 \\
\hline 8 & 0 & 75 & No & No & 51 & 0 & 0 & 0 & 0 \\
\hline 12 & $\mathrm{O}$ & 91 & No & No & 59 & 0 & 0 & 0 & 0 \\
\hline 13 & $\mathrm{H}$ & 1100 & Yes & Yes & 41 & 32 & 35 & 22 & 26 \\
\hline $1,3-7,9^{++}$ & $\mathrm{C}$ & $\mathrm{N}$ & No & No & & 0 & 0 & 0 & 0 \\
\hline
\end{tabular}

* $\mathrm{O}=$ Obligate carrier, $\mathrm{H}=$ High probability carrier, $\mathrm{C}=\mathrm{Control}$ subject.

+Normal range: $28-110 \mathrm{U} / \mathrm{L} ; \mathrm{N}=$ normal.

$+{ }^{+}$The seven control subjects are numbered 1, 3-7,9. 
$\mathrm{X}$-inactivation, regional clustering of dystrophin-incompetent nuclei, fusion with dystrophin-competent satellite cells, selection against dystrophin-negative fibres and the length of membrane for which a nucleus can provide the genetic information for dystrophin. The detection of a mosaic staining pattern in both human and $\mathrm{mdx}$ mouse heterozygotes (data not shown) confirms the presence of Lyonization although little can be definitively concluded from our study regarding the pathogenesis of this variability.

The results of our blind study clearly indicate that immunohistochemistry is specific enough to be used as a diagnostic test. Our study however, fails to reach a definitive conclusion on the sensitivity of the test. Although $4 / 6$ carriers were detected, two of these biopsies had negative fibres present at such low frequency that technical and sampling errors might have resulted in the negative fibres being missed. Perhaps counting more than three hundred fibres at multiple levels from the same biopsy may increase the sensitivity of the test.

A comparison of our results using various combinations of antibodies and protocols identified the use of the monoclonal antibody Dys 2 and immunofluorescence as the most sensitive

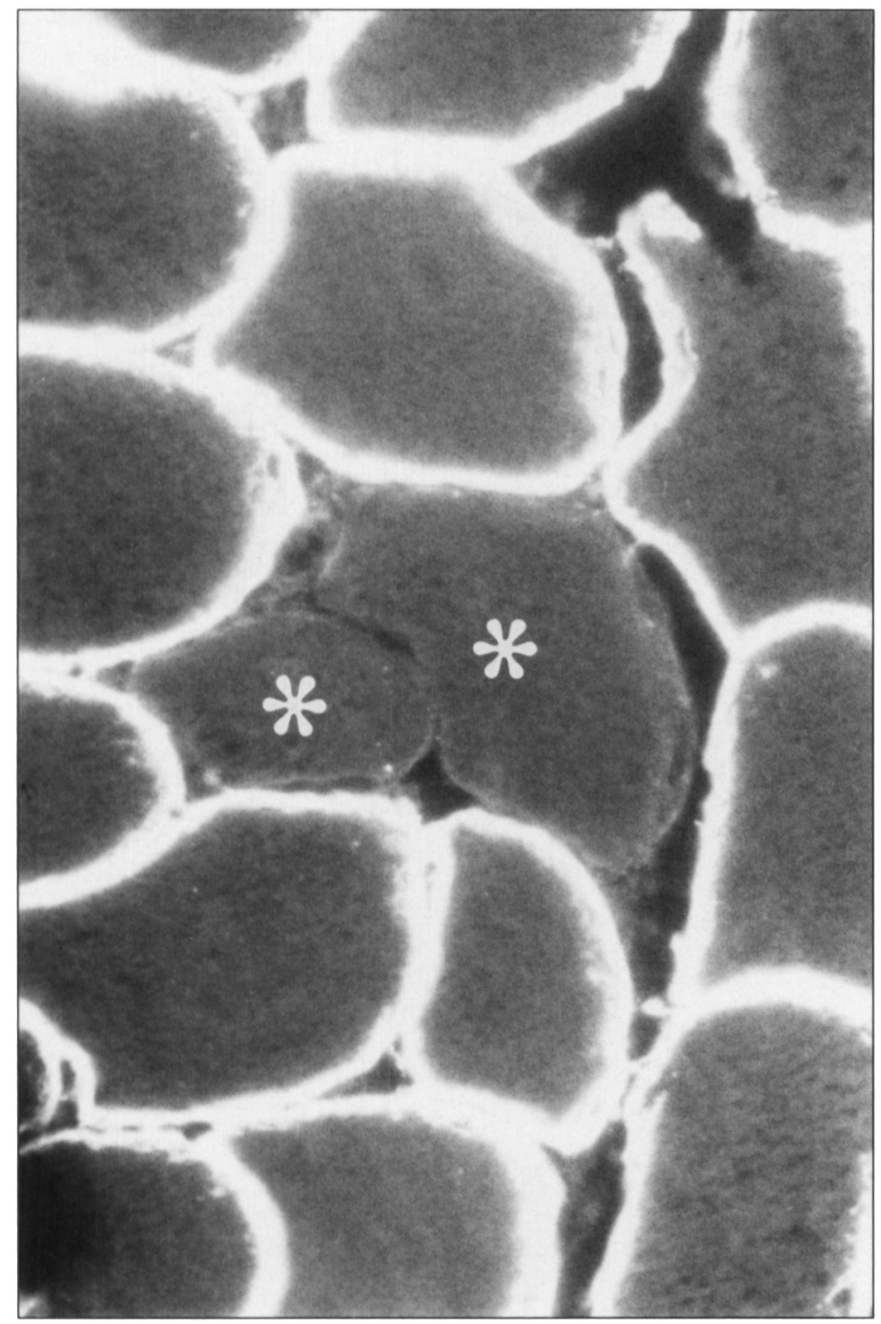

Figure I - Cross-section of a muscle biopsy from a DMD carrier: Indirect immunofluorescence shows both dystrophin-positive and dystrophin-negative fibres (asterisks). Magnification $\times 192$. combination (Table 1). Dys 1 failed to identify 3 of 6 carriers and the peroxidase technique failed to identify 4 of 6 carriers. Although sampling errors can account for this discrepancy, the monoclonal antibody Dys 2 in combination with immunofluorescence gave much cleaner preparations which were easier to score.

The presence of fibres scored as uncertain was felt to be largely due to technical factors and mainly involved the peroxidase technique. No significance or pattern could be attributed to the presence or proportion of uncertain fibres.

Although the prospect of assigning a high probability carrier status based on the presence of only a few negative fibres is daunting, we and several other groups have never identified a negative fibre in a normal control ${ }^{2-6}$. We therefore conclude that the presence of negative fibres in any proportion is significant and diagnostic although the absence of negative fibres is not sufficient to exclude the diagnosis.

Several previous reports have indicated that dystrophin immunohistochemistry can detect some carriers with high CK values $^{2.3 .11,12}$. All four of the biopsies with negative fibres in our study were from carriers with elevated CK levels while the two carriers with normal CK went undetected. However, CK levels may also vary in normal individuals following exercise, with age and in pregnancy..$^{13-15}$ Hääriäinen et al. ${ }^{16}$ described five families in whom a sister or maternal aunt had elevated CK activity although DNA analysis predicted low risk DMD carrier status. We have also encountered similar situations and conclude that CK values, due to their non-specific nature, remain difficult to interpret in certain situations and that dystrophin immunohistochemistry is an important adjunct.

The presence of an age-related conversion of negative-fibres to positive fibres in heterozygote $\mathrm{mdx}$ mice as initially reported by Karpati et al. ${ }^{17}$ was confirmed in our own lab (data not shown). These results may explain the observed phenomena in women whereby $\mathrm{CK}$ levels are higher in younger carrier women but tend to fall in later years. ${ }^{15.16}$ Practically, the phenomenon seen in mice suggests that immunohistochemistry may be more sensitive in younger women and may need to be performed during adolescence or childhood to have its greatest diagnostic sensitivity. It is interesting to note that the two subjects in our study who did not have negative fibres were also the two oldest women ( $51 \& 59 \mathrm{yrs})$ in our study. Although Vainzof et al..$^{9} \mathrm{did}$ not find this correlation between negative fibres and young asymptomatic carriers, the benefits, role and diagnostic value of testing younger women should be further carefully explored. In addition, the recent report by Mateddu et al. ${ }^{7}$ demonstrates variable dystrophin expression between different muscle groups of DMD carriers implying immunohistochemical characterization of dystrophin expression in more than one muscle may improve the sensitivity of this analysis.

We believe that immunohistochemistry will prove to be useful in DMD families where no DNA is available or as an adjunct to $C K$ values. However, further studies are needed to determine the sensitivity of immunohistochemistry and whether immunohistochemical analysis will prove to be more valuable in greater than one muscle and in younger females.

\section{ACKNOWLEDGEMENTS}

We wish to acknowledge the assistance of Dr. Ray Postuma, pediatric surgeon, Childrens' Hospital, Winnipeg, who performed all the 
muscle biopsies, and the DMD carrier females who willingly participated in this study. The financial support of the Muscular Dystrophy Association of Canada and the Children's Hospital of Winnipeg Research Foundation is gratefully acknowledged.

\section{REFERENCES}

1. Haldane JBS. The rate of spontaneous mutation of a human gene. J Genet 1935; 31: 317-326.

2. Arahata K, Hoffman EP, Kunkel LM, et al. Dystrophin diagnosis: comparison of dystrophin abnormalities by immunofluorescence and immunoblot analyses. Proc Natl Acad Sci USA 1989; 86: 7154-7158.

3. Arahata K, Ishihara T, Kamakura K, et al. Mosaic expression of dystrophin in symptomatic carriers of Duchenne's muscular dystrophy. N Engl J Med 1989; 320: 138-142.

4. Bonilla E, Schmidt B, Samitt CE, et al. Normal and dystrophindeficient muscle fibres in carriers of the gene for Duchenne muscular dystrophy. Am J Pathol 1988; 133: 440-445.

5. Morandi L, Mora M. Gussoni E, et al. Dystrophin analysis in Duchenne and Becker muscular dystrophy carriers: correlation with intracellular calcium and albumin. Ann Neurol 1990; 28 674-679.

6. Hoffman EP, Arahata K, Minetti C, et al. Dystrophinopathy in isolated cases of myopathy in females. Neurology 1992; 42: 967 975.

7. Mateddu MF, Marrosu MG, Cau M, et al. Variable dystrophin expression in different muscles of a Duchenne muscular dystrophy carrier. Clin Genet 1992; 42: 35-38.

8. Clerk A, Rodillo E, Heckmatt JZ et al. Characterization of dystrophin in carriers of Duchenne muscular dystrophy. J Neurol Sci 1991; 102: 197-205.
9. Vainzof M, Pavanello RCM, Pavanello I, el al. Dystrophin immunofluorescence pattern in manifesting and asymptomatic carriers of Duchenne's and Becker muscular dystrophies of different ages. Neuromuscular Dis 1991; 1: 177-183.

10. Gartler SM, Riggs AD. Mammalian X-chromosome inaclivation. Ann Rev Genet 1983; 17: 155-190.

11. Hoffman EP, Fischbeck KH, Brown RH, et al. Characterization of dystrophin in muscle-biopsy specimens from patients with Duchenne's or Becker's muscular dystrophy. N Engl J Med 1988; 318: 1363-1368.

12. Shumate JB, Brooke MH, Carroll JE, Davis JE.1979. Increased serum creatine kinase after exercise: a sex-linked phenomenon. Neurology 1979; 29: 902-904.

13. Perry TB, Clarke F. Variability of serum creatinine phosphokinase activity in normal women and carriers of the gene for Duchenne muscular dystrophy. Neurology 1973; 23: 1316-1323.

14. Bundley S, Crawley JM, Edwards JH, Westhead RA. Serum creatine kinase levels in pubertal, mature, pregnant and postmenopausal women. J Med Genet 1979; 16:117-121.

15. Moser $\mathrm{H}$. Duchenne muscular dystrophy: pathogenetic aspects and genetic prevention. Hum Genet 1984; 66: 17-40.

16. Hääriäinen H, Lindlof M, Somer H, DeLaChapelle A. Genetic counselling in Duchenne and Becker muscular dystrophy is problematic when carrier studies give controversial results. Clin Genet 1990; 37: 179-187.

17. Karpati G, Zubrzycka-Gaarn E, Carpenter S, et al. Age-related conversion of dystrophin-negative to -positive fibre segments of skeletal but not cardiac muscle fibres in heterozygote mdx mice. J Neuropathol Exp Neurol 1990; 49: 96-105. 